\title{
A Hopf algebra of subword complexes (Extended abstract)
}

\author{
Nantel Bergeron $\rrbracket^{1}$ and Cesar Ceballos 2 ” \\ ${ }^{1}$ Department of Mathematics and Statistics, York University, Canada \\ ${ }^{2}$ Faculty of Mathematics, University of Vienna, Austria.
}

\begin{abstract}
We introduce a Hopf algebra structure of subword complexes, including both finite and infinite types. We present an explicit cancellation free formula for the antipode using acyclic orientations of certain graphs, and show that this Hopf algebra induces a natural non-trivial sub-Hopf algebra on $c$-clusters in the theory of cluster algebras.

Résumé. Nous introduisons une structure d'algèbre de Hopf sur les complexes de sous-mots de types fini et infini. Nous présentons une formule explicite sans cancelation pour l'antipode qui utilise les orientations acycliques. Nous donnons une sous-algèbre de Hopf naturel non-trivial sur les $C$-clusters dans la théorie des algèbres amassées.
\end{abstract}

Keywords. Subword complexes, Hopf algebras, antipode, cluster algebras

\section{Introduction}

Subword complexes are simplicial complexes introduced by Knutson and Miller, and are motivated by the study of Gröbner geometry of Schubert varieties [14, 15]. These complexes have been shown to have striking connections with diverse topics such as associahedra [16], multi-associahedra [13], pseudotriangulation polytopes [21], and cluster algebras [9].

The first connection between subword complexes and associahedra was discovered by Pilaud and Pocchiola who showed that every multi-associahedron can be obtained as a well chosen type $A$ subword complex in the context of sorting networks [17]. These results were generalized to arbitrary finite Coxeter groups by Ceballos, Labbé and Stump in [4]. The results in [4] provide an additional connection with the $c$-cluster complexes in the theory of cluster algebras, which has been used as a keystone for explicit results about denominator vectors in cluster algebras of finite type [5]. A construction of certain brick polytopes of spherical subword complexes is presented in [18, 19], which is used to give a precise description of the toric varieties of $c$-generalized associahedra in connection with Bott-Samelson varieties in [7]. More recent developments on geometric and combinatorial properties of subword complexes are presented in [3, 8, 23].

\footnotetext{
†Email: bergeroneyorku.ca. Partially supported by NSERC.

‡Email: cesar.ceballos@univie.ac.at. Partially supported by the government of Canada through a Banting Postdoctoral Fellowship, by a York University research grant, and by the Austrian Science Foundation FWF, grant F 5008-N15, in the framework of the Special Research Program "Algorithmic and Enumerative Combinatorics".
} 
This paper presents a more algebraic approach to subword complexes. We introduce a Hopf algebra structure on the vector space generated by all facets of irreducible subword complexes, including both finite and infinite types. Such facets include combinatorial objects such as triangulations and multitriangulations of convex polygons, pseudotriangulations of any planar point set in general position, and $c$-clusters in cluster algebras of finite type. We present an explicit cancellation-free formula for the antipode using acyclic orientations of certain graphs. It is striking to observe that we obtain a result very similar to the antipode formula of Humpert and Martin for the incidence Hopf algebra of graphs [12]. As in [2], our combinatorial Hopf algebra is part of a nice family with explicit cancelation-free formula for the antipode. The Hopf algebra of subword complexes also induces a natural sub-Hopf algebra on $c$-clusters of finite type. Cluster complexes for Weyl groups were introduced by Fomin and Zelevinsky in connection with their proof of Zamolodchikov's periodicity conjecture for algebraic $Y$-systems in [10]. These complexes encode the combinatorial structure behind the associated cluster algebra of finite type [9], and are further extended to arbitrary Coxeter groups by Reading in [20]. The resulting $c$-cluster complexes use a Coxeter element $c$ as a parameter and have been extensively used to produce geometric constructions of generalized associahedra [11, 22, 19]. The basis elements of our Hopf algebra of $c$-clusters are given by pairs of clusters $(A, T)$ of finite type, where $A$ is any acyclic cluster seed and $T$ is any cluster obtained from it by mutations. The multiplication and comultiplication operations are natural from the cluster algebra perspective on $T$. However, subword complexes allow us to nontrivially extend these operations to remarkable operations on the acyclic seed $A$.

The outline of the paper is as follows: In Section 2 we present the concept of subword complexes, some examples and a decomposition theorem needed for the Hopf algebra structure. In Section 3 we give the Hopf structure and compute explicitly a cancelation-free formula for the antipode. In Section 4 we show that this Hopf algebra induces a sub-Hopf algebra on $c$-clusters of finite type and present a combinatorial model description for Cartesian products of classical types. The full paper (31 pages) is available on ArXive1508.01465, here we omit the proofs, many of the comments and some other features. In particular, our theorems and constructions work for infinite Coxeter groups as well.

\section{Subword Complexes}

Let $W$ be a possibly infinite Coxeter group with generators $S=\left\{s_{1}, \ldots, s_{n}\right\}$. This group acts on a real vector space $V$, we denote by $\Delta:=\left\{\alpha_{s} \mid s \in S\right\}$ the set of simple roots of a root system $\Phi=\Phi^{+} \sqcup \Phi^{-}$ associated to $W$. Throughout the paper, for simplicity, we think of $W$ as the tuple $\left(W, S, \Phi^{+}\right)$containing the information of the group, its generators and the decomposition of its root system $\Phi=\Phi^{+} \sqcup-\left(\Phi^{+}\right)$.

Definition 2.1 ([14]) Let $Q=\left(q_{1}, \ldots, q_{r}\right)$ be a word in $S$ and $\pi \in W$ be an element of the group. The subword complex $\mathcal{S C}(Q, \pi)$ is a simplicial complex whose faces are given by subsets $I \subset[r]=$ $\{1,2, \ldots, r\}$, such that the subword of $Q$ with positions at $[r] \backslash I$ contains a reduced expression of $\pi$.

Example 2.2 Let $W=\mathcal{S}_{3}$ be the symmetric group generated by the simple transpositions $S=\left\{s_{1}, s_{2}\right\}=$ $\{(12),(23)\}$. Let $Q=\left(q_{1}, q_{2}, q_{3}, q_{4}, q_{5}\right)=\left(s_{1}, s_{2}, s_{1}, s_{2}, s_{1}\right)$ and $\pi=s_{1} s_{2}$. Since the reduced expressions of $\pi$ in $Q$ are given by $q_{1} q_{2}=q_{1} q_{4}=q_{3} q_{4}=\pi$, the maximal faces of $\mathcal{S C}(Q, \pi)$ are $\{3,4,5\},\{2,3,5\}$ and $\{1,2,5\}$. This subword complex is illustrated in Figure 1 , where we use the network diagrams used by Pilaud and Pocchiola in [17]. Such diagrams will be used through out the paper to represent subword complexes of type A. The letters in the word $Q$ are consecutively placed form left to 
right as vertical commutators in the diagram such that a generator $s_{i}$ connects the horizontal levels $i$ and $i+1$ numerated from bottom to top.
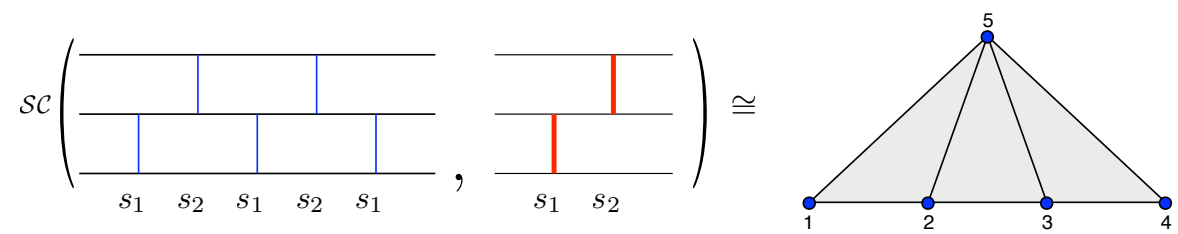

Fig. 1: Subword complex $\mathcal{S C}(Q, \pi)$ for $Q=\left(s_{1}, s_{2}, s_{1}, s_{2}, s_{1}\right)$ and $\pi=s_{1} s_{2}$ in type $A_{2}$.

Two remarkable examples of subword complexes are the dual associahedron and multi-associahedron. The first description of these two complexes as well chosen subword complexes was given by Pilaud and Pocchiola in the context of sorting networks in [17, Section 3.3 and Theorem 23].

Example 2.3 (Multi-associahedron) Let $W=\mathcal{S}_{4}$ and $S=\left\{s_{1}, s_{2}, s_{3}\right\}=\{(12),(23),(34)\}$ as above, $Q=\left(s_{1}, s_{2}, s_{3}, s_{1}, s_{2}, s_{3}, s_{1}, s_{2}, s_{3}, s_{1}, s_{2}, s_{1}\right)$ and $\pi=\left[\begin{array}{lll}4 & 3 & 2\end{array}\right]$. The subword complex $\mathcal{S C}(Q, \pi)$ is isomorphic to the (simplicial) multi-associahedron $\Delta_{8,2}$. The vertices of this complex are the 2-relevant diagonals of a convex 8-gon, that is diagonals that leave at least two vertices of the polygon on each of its sides. The faces are subsets of 2-relevant diagonals not containing a 3-crossing, that is 3 diagonals that mutually cross in their interiors. The thick blue diagonals in the right part of Figure 2 form a maximal set of 2-relevant diagonals not containing a 3-crossing. The corresponding facet $I=\{1,3,7,8,9,10\}$ of the subword complex is illustrated on the left. The bijection sends the ith letter in $Q$ to the ith 2-relevant diagonal of the polygon in lexicographic order. Note that the thin grey diagonals in the figure never appear in a 3-crossing and therefore are considered to be "irrelevant". A maximal set of diagonals (relevant or not) of a polygon not containing a $(k+1)$-crossing is known in the literature as a $k$-triangulation. We refer to [4] Section 2] for more details on this bijection in the generality of [17].

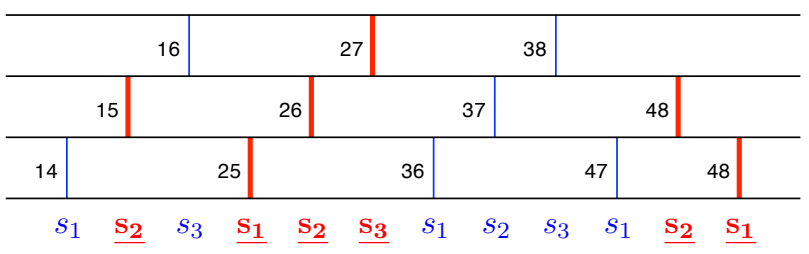

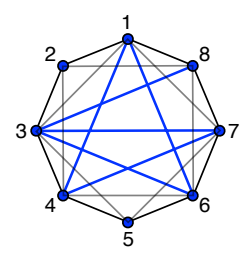

Fig. 2: Bijection between the facets of the illustrated subword complex and 2-triangulations of a convex 8-gon.

The multi-associahedron is a rich combinatorial object which is conjectured to be realizable as the boundary complex of a convex polytope [13, Section 1.2]. Inspired by our Hopf algebra of subword complexes, we discovered certain geometric constructions of a particular family of multi-associahedra [3]. Another important family of examples in connection with cluster complexes in cluster algebras, and the corresponding induced Hopf algebra will be presented in Section 4 


\subsection{Root function and flats}

Associated to a subword complex, one can define a root function which plays a fundamental role in the theory. This function was introduced by Ceballos, Labbé and Stump in [4]. It encodes exchanges in the facets of the subword complex [4] and has been extensively used in the construction of Coxeter brick polytopes [19] and in the description of denominator vectors in cluster algebras of finite type [5].

Definition 2.4 ([4] $)$ The root function $\mathrm{r}(I, \cdot):[r] \longrightarrow \Phi$ associated to a subset $I \subseteq[r]$ is defined by

$$
\mathrm{r}(I, j):=\sigma_{A_{j}}\left(\alpha_{q_{j}}\right),
$$

where $A_{j}:=[j-1] \backslash I$ is the set of positions on the left of $j$ that are in the complement of $I$, and $\sigma_{X} \in W$ denotes the product of the elements $q_{x} \in Q$ for $x \in X$ in the order they appear in $Q$. The root configuration of $I$ is the list $\mathrm{R}(I):=(\mathrm{r}(I, i): i \in I)$. We denote by $\mathrm{r}(I, Q)$ the list of roots $(\mathrm{r}(I, 1), \ldots, \mathrm{r}(I, r))$.

All the information about the subword complex is encoded by its root function. In particular, the flips between facets can be described as follows. Lemma 2.5 was stated for subword complexes of finite type in [4], but the proof works exactly the same for arbitrary Coxeter groups (finite or not).

Lemma 2.5 ([4, Lemmas 3.3 and 3.6]) Let $I$ and $I^{\prime}$ be two adjacent facets of the subword complex $\operatorname{SC}(Q, \pi)$ with $I \backslash i=I^{\prime} \backslash i^{\prime}$.

(1) The position $i^{\prime}$ is the unique position in the complement of I such that $\mathrm{r}\left(I, i^{\prime}\right) \in\{ \pm \mathrm{r}(I, i)\}$. Moreover, $\mathrm{r}\left(I, i^{\prime}\right)=\mathrm{r}(I, i)$ if $i<i^{\prime}$, while $\mathrm{r}\left(I, i^{\prime}\right)=-\mathrm{r}(I, i)$ if $i^{\prime}<i$.

(2) The map $\mathrm{r}\left(I^{\prime}, \cdot\right)$ is obtained from the map $\mathrm{r}(I, \cdot)$ by

$$
\mathrm{r}\left(I^{\prime}, k\right)= \begin{cases}s_{\mathrm{r}(I, i)} \mathrm{r}(I, k) & \text { if } \min \{i, j\}<k \leq \max \{i, j\} \\ \mathrm{r}(I, k) & \text { otherwise }\end{cases}
$$

where $s_{\mathrm{r}(I, i)} \in W$ denotes the reflection that is orthogonal (or dual) to the root $\mathrm{r}(I, i)$.

Example 2.6 (Example 2.3 continued) Let $\left\{\alpha_{1}, \ldots, \alpha_{n}\right\}$ be the simple roots of the root system of type $A_{n}$. The positive roots can be written as positive linear combinations $\alpha_{i \ldots j}=\sum_{\ell=i}^{j} \alpha_{\ell}$, for $1 \leq i \leq j \leq n$, and the negative roots are the roots $-\alpha_{i \ldots j}$. The group acts on the roots according to the following rule which is extended by linearity,

$$
s_{i}\left(\alpha_{j}\right)= \begin{cases}-\alpha_{j} & \text { if } i=j \\ \alpha_{i}+\alpha_{j} & \text { if }|i-j|=1 \\ \alpha_{j} & \text { otherwise. }\end{cases}
$$

The root function of the subword complex in Example 2.3 with respect to the facet $I=\{1,3,7,8,9,10\}$ is illustrated in Figure 3 It associates a root to each of the letters in the word, the root $\alpha_{i \ldots j}$ would be represented in the diagram by the indices $i . . . j$ for simplicity. For example, the indices 23 represent the root $\alpha_{23}=\alpha_{2}+\alpha_{3}$. Indices corresponding to diagonals of a polygon are placed on the left of each commutator, while indices corresponding to roots are placed on the right throughout the paper. The root associated to a letter $s_{j}$ in $Q$ can be thought as the underlined red word on the left of that letter applied to $\alpha_{j}$. 


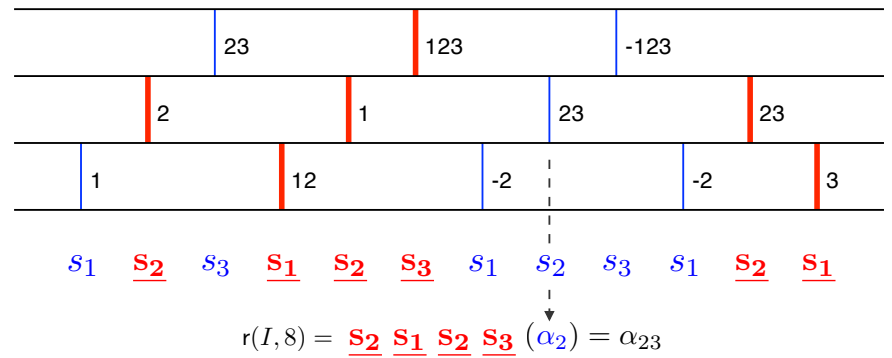

Fig. 3: Root function of the subword complex in Example 2.3 for the facet $I=\{1,3,7,8,9,10\}$.

Note that exchanges in facets can be easily performed knowing the root function. For example, any of the two thin blue commutators labeled 23 can be flipped to the unique bold red commutator 23 to form a new facet. Similarly, any 2-relevant blue diagonal in the 2-triangulation in Figure 2 can be flipped to a unique diagonal to form a new 2-triangulation. However such flips are much easier to visualize in the subword complex.

Definition 2.7 A subword complex $\mathcal{S C}(Q, \pi)$ is said to be irreducible if and only if the root configuration $\mathrm{R}(I)$ generates the vector space $V$ for some facet $I$. Or equivalently, if the root configuration $\mathrm{R}(I)$ generates the vector space $V$ for any facet $I$ (these two conditions are equivalent by Lemma 2.5 2) and the fact that any two facets are connected by a sequence of flips). A non irreducible subword complex is called reducible.

We will see below that every reducible subword complex is isomorphic to a subword complex of smaller rank (Corollary 2.11). This explains our choice of terminology. Before proving this, we need a notion of flats of a list of vectors in a vector space.

Definition 2.8 Let $L=\left(v_{1}, \ldots, v_{r}\right)$ be a list of vectors (with possible repetitions) spanning a vector space $V$. A flat $F$ of $L$ is any sublist $F \subset L$ that can be obtained as the intersection $F=U \cap L$ for some subspace $U \subset V$.

The flats of $r(I, Q)$ will be used to define the comultiplication of the Hopf algebra structure on subword complexes. The main ingredient in the definition is that every flat encodes the root function of a subword complex of smaller rank, which turns out to be isomorphic to the link of a face of the initial subword complex. This result, which we call the "Decomposition theorem of subword complexes", has its origins in [4] and was presented for finite types in a slightly weaker version in [19].

\subsection{Decomposition theorem of subword complexes}

Given a flat $F$ of $r(I, Q)$ denote by $V_{F} \subset V$ the subspace of $V$ spanned by the roots in $F$. This subspace contains a natural root system

$$
\Phi_{F}=\Phi_{F}^{+} \sqcup \Phi_{F}^{-}
$$

where $\Phi_{F}, \Phi_{F}^{+}, \Phi_{F}^{-}$are the restrictions of $\Phi, \Phi^{+}, \Phi^{-}$to $V_{F}$ respectively. We denote by $\Delta_{F}$ the corresponding set of simple roots and by $W_{F}$ the associated Coxeter group. In the case of infinite Coxeter 
groups, the fact that the root system intersected with a subspace is again a root system with simple roots contained in $\Phi^{+}$is a non-trivial result by Dyer in [6]. For convenience, denote by

$$
J_{F}=\left\{j_{1}, \ldots, j_{r^{\prime}}\right\} \subset[r]
$$

the set of positions in $Q$ whose corresponding roots $r\left(I, j_{k}\right)$ belong to $F$. Define $\beta_{F}=\left(\beta_{1}, \ldots, \beta_{r^{\prime}}\right)$ as the list of roots

$$
\beta_{k}:=\sigma_{B_{j_{k}}}\left(\alpha_{q_{j_{k}}}\right)
$$

where $B_{j}:=([j-1] \backslash I) \backslash J_{F}$ is the set of positions on the left of $j$ in the complement of $I$ whose corresponding roots are not in $F$.

Lemma 2.9 The roots $\beta_{1}, \ldots, \beta_{r^{\prime}}$ are simple roots of the root system $\Phi_{F}$.

We will define a subword complex $\mathcal{S C}\left(Q_{F}, \pi_{F}\right)$ and a facet $I_{F}$ associated to $F$. Denote by

$$
Q_{F}:=\left(q_{1}^{\prime}, \ldots, q_{r^{\prime}}^{\prime}\right)
$$

the word whose letters are the generators of the Coxeter group $W_{F}$ corresponding to the simple roots $\beta_{1}, \ldots, \beta_{r^{\prime}}$. The set $I_{F}$ corresponding to $I$ is given by

$$
I_{F}:=\left\{i \in\left[r^{\prime}\right]: j_{i} \in I\right\}
$$

and the element $\pi_{F} \in W_{F}$ is the product of the letters in the subword of $Q_{F}$ with positions at the complement of $I_{F}$. We also denote by $\bar{I}_{F}$ the face of $\mathcal{S C}(Q, \pi)$ corresponding to $I_{F}$, or in other words, the elements $i \in I$ whose corresponding roots $\mathrm{r}(I, i)$ belong to $F$.

Theorem 2.10 (Decomposition theorem of subword complexes) Let I be a facet of a subword complex $\mathcal{S C}(Q, \pi)$ of (possibly infinite) type $W$. If $F$ is a flat of $\mathrm{r}(I, Q)$, then $F$ is the root function of the subword complex $\mathcal{S C}\left(Q_{F}, \pi_{F}\right)$ of type $W_{F}$ with respect to the facet $I_{F}$. Moreover,

$$
\mathcal{S C}\left(Q_{F}, \pi_{F}\right) \cong \operatorname{Link}_{\mathcal{S C}(Q, \pi)}\left(I \backslash \bar{I}_{F}\right)
$$

Corollary 2.11 A reducible subword complex is isomorphic to an irreducible subword complex of smaller rank. (also see [19. Proposition 3.6] and [18. Proposition 3.7])

Example 2.12 (Example2.3 continued) Consider the subword complex in Example 2.3 and the root function associated to the facet $I=\{1,3,7,8,9,10\}$ (also illustrated in Figure 3):

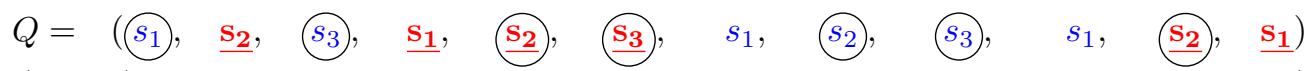

$$
\begin{aligned}
& \mathrm{r}(I, Q)=\left(\alpha_{1}, \quad \underline{\alpha_{2}}, \quad \alpha_{23}, \quad \underline{\alpha_{12}}, \quad \underline{\alpha_{1}}, \quad \underline{\alpha_{123}}, \quad-\alpha_{2}, \quad \alpha_{23}, \quad-\alpha_{123}, \quad-\alpha_{2}, \quad \underline{\alpha_{23}}, \quad \underline{\alpha_{3}}\right)
\end{aligned}
$$

Let $F=\left(\alpha_{1}, \alpha_{23}, \alpha_{1}, \alpha_{123}, \alpha_{23},-\alpha_{123}, \underline{\alpha_{23}}\right)$ be the flat at positions $J_{F}=\{1,3,5,6,8,9,11\}$. The list of beta simple roots and the associated word are

$$
\begin{aligned}
& \beta_{F}=\left(\begin{array}{lllllll}
\alpha_{1}, & \alpha_{23}, & \underline{\alpha_{1}}, & \underline{\alpha_{23}}, & \alpha_{1}, & \alpha_{23}, & \underline{\alpha_{1}}
\end{array}\right) \\
& Q_{F}=
\end{aligned}
$$

There is one root in $\beta_{F}$ for each circled letter $s_{j}$ in $Q$. This root is computed by applying all the underlined red letters which are not circled on its left to $\alpha_{j}$. For example, for the fourth circled letter, which is an $s_{3}$ 
in this case, one gets the root $\beta_{4}=\underline{\mathrm{s}_{2} \mathrm{~s}_{1}}\left(\underline{\alpha_{3}}\right)=\underline{\alpha_{23}}$. The restricted facet is $I_{F}=\{1,2,5,6\}$ and the element $\pi_{F}=s_{1} s_{23} s_{1}$. The Coxeter group $W_{F}$ is generated by the simple transpositions $\left\{s_{1}, s_{23}\right\}$, and turns out to be isomorphic to the symmetric group $\mathcal{S}_{3}$. Thus, $\mathcal{S C}\left(Q_{F}, \pi_{F}\right)$ can be written as the type $A_{2}$ subword complex

$$
\mathcal{S C}\left(Q_{F}, \pi_{F}\right)=\mathcal{S C}\left(\left(s_{1}, s_{2}, s_{1}, s_{2}, s_{1}, s_{2}, s_{1}\right),\left[\begin{array}{lll}
3 & 2 & 1
\end{array}\right]\right)
$$

The tuple $\left(W_{F}, Q_{F}, \pi_{F}, I_{F}\right)$ is called a flat decomposition of $(W, Q, \pi, I)$.

\section{A Hopf algebra of subword complexes}

Let $Y_{n}$ be the set of equivalent classes of tuples $(W, Q, \pi, I)$ where $W$ is a (possibly infinite) Coxeter group of rank $n$, and $I$ is a facet of an irreducible subword complex $\mathcal{S C}(Q, \pi)$. For $n=0$, by convention, we assume there is a unique empty tuple $\mathbf{1}=\left(W_{0}, \emptyset, \emptyset, \emptyset\right)$ and in particular $\left|Y_{0}\right|=1$. Two tuples are considered to be equivalent, denoted by $(W, Q, \pi, I) \cong\left(W^{\prime}, Q^{\prime}, \pi^{\prime}, I^{\prime}\right)$, if and only if there is an isomorphism $\phi: W \rightarrow W^{\prime}$ which maps generators of $W$ to generators of $W^{\prime}$ such that $\phi(Q)=Q^{\prime}$ up to commutation of consecutive commuting letters, $\phi(\pi)=\pi^{\prime}$ and $I^{\prime}$ are the positions in $Q^{\prime}$ that correspond (up to the performed commutations) to the positions of $I$ in $\phi(Q)$. Note that such commutations only alter the subword complexes by relabelling of its vertices.

The main result of this section is to show that the graded vector space

$$
\mathcal{Y}:=\bigoplus_{n \geq 0} k\left[Y_{n}\right]
$$

may be equipped with a structure of connected graded Hopf algebra. We recommend the reader to [1] for more on connected graded Hopf algebra's axioms.

Remark 3.1 Note that $k\left[Y_{n}\right]$ is infinite dimensional. In most situations, we need finite dimensional subspaces compatible with the Hopf structure. For this, we introduce a double filtration of the spaces $k\left[Y_{n}\right]$. Let

$$
k\left[Y_{n}\right]=\bigcup_{\substack{m \geq 2 \\ \ell \geq 1}} k\left[Y_{n}^{m, \ell}\right]
$$

where $Y_{n}^{m, \ell}$ is the set of equivalent classes of tuples $(W, Q, \pi, I)$ such that $W$ is a of rank $n, Q$ is of length $\leq \ell$, and for any two generators $s_{i}, s_{j} \in S$, the smallest $m_{i j}$ such that $\left(s_{i} s_{j}\right)^{m_{i j}}=1$ satisfies $m_{i j} \leq m$ or $m_{i j}=\infty$. We now have that $Y_{n}^{m, \ell}$ is finite, hence $k\left[Y_{n}^{m, \ell}\right]$ is finite dimensional.

\subsection{Comultiplication $\Delta: \mathcal{Y} \rightarrow \mathcal{Y} \otimes \mathcal{Y}$ and counit $\epsilon: \mathcal{Y} \rightarrow k$.}

Definition 3.2 Let $V$ denote the space generated by $\mathrm{R}(I)$. A k-flat-decomposition of $\mathrm{R}(I)$ is a $k$-tuple of flats $\left(F_{1}, F_{2}, \ldots, F_{k}\right)$ such that the $F_{i}$ 's are irreducible flats of $\mathrm{r}(I, Q)$, that is the space $V_{F_{i}}$ spanned by $F_{i}$ is the same as the space spanned by the roots in $\mathrm{R}\left(I_{F_{i}}\right)$, and we also require that $V=V_{F_{1}} \oplus V_{F_{2}} \oplus$ $\cdots \oplus V_{F_{k}}$.

Definition 3.3 The subword complex comultiplication of a tuple $(W, Q, \pi, I)$ is defined as

$$
\Delta(W, Q, \pi, I):=\sum_{\left(F_{1}, F_{2}\right)}\left(W_{F_{1}}, Q_{F_{1}}, \pi_{F_{1}}, I_{F_{1}}\right) \otimes\left(W_{F_{2}}, Q_{F_{2}}, \pi_{F_{2}}, I_{F_{2}}\right),
$$


where the sum is over all 2-flat-decompositions $\left(F_{1}, F_{2}\right)$ of $\mathrm{R}(I)$. The map $\Delta$ is then extended to $\mathcal{Y}$ by linearity. We have

$$
\Delta: k\left[Y_{n}^{m, \ell}\right] \longrightarrow \bigoplus_{\substack{n_{1}+n_{1}=n \\ \ell_{1}+\ell_{2}=\ell}} k\left[Y_{n_{1}}^{m, \ell_{1}}\right] \otimes k\left[Y_{n_{2}}^{m, \ell_{2}}\right] .
$$

The counit for $\Delta$ is given by $\epsilon: \mathcal{Y} \rightarrow k$ where $\epsilon\left(Y_{n}\right)=0$ for $n>0$ and $\epsilon(\mathbf{1})=1$. This map clearly satisfies the axioms of a counit. This makes $(\mathcal{Y}, \Delta, \epsilon)$ a graded cocommutative coalgebra.

Example 3.4 (Example 2.2 continued) The comultiplication of the facet $I=\{2,3,5\}$ of the subword complex $\mathcal{S C}\left(\left(s_{1}, s_{2}, s_{1}, s_{2}, s_{1}\right), s_{1} s_{2}\right)$ in Example 2.2 is given in Figure 4

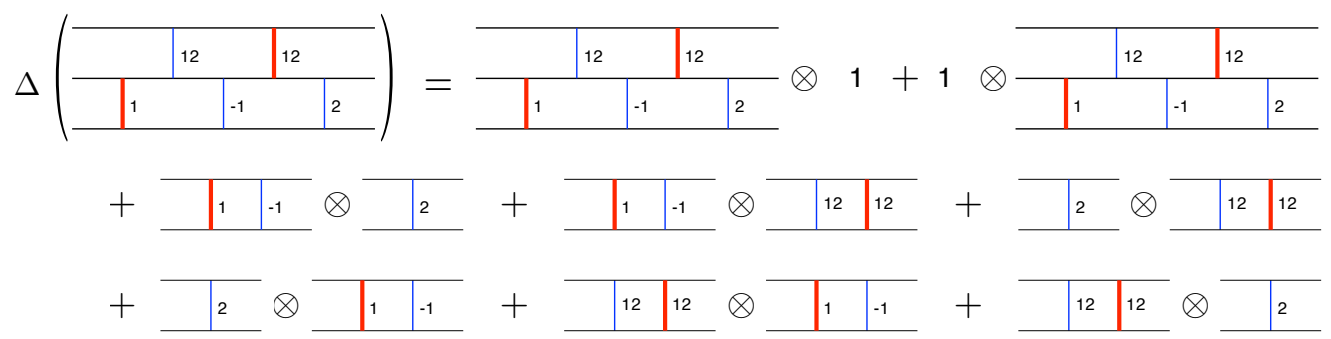

Fig. 4: Example of the subword complex comultiplication.

\subsection{Multiplication $m: \mathcal{Y} \otimes \mathcal{Y} \rightarrow \mathcal{Y}$ and unit $u: k \rightarrow \mathcal{Y}$.}

Let $W$ and $W^{\prime}$ be two Coxeter groups with generating sets $S=\left\{s_{1}, \ldots, s_{n}\right\}$ and $S^{\prime}=\left\{s_{1}^{\prime}, \ldots, s_{n}^{\prime}\right\}$ respectively. Let $\Phi$ and $\Phi^{\prime}$ be two associated root systems with simple roots $\Delta=\left\{\alpha_{s} \mid s \in S\right\}$ and $\Delta^{\prime}=\left\{\alpha_{s^{\prime}} \mid s^{\prime} \in S^{\prime}\right\}$. We denote by $\bar{W}=W \times W^{\prime}$ the augmented Coxeter group generated by the disjoint union $S \sqcup S^{\prime}$, where the generators of $S$ are set to commute with all the generators of $S^{\prime}$. In other words, the Coxeter graph of $\bar{W}$ is the union of the Coxeter graphs of $W$ and $W^{\prime}$. The corresponding augmented root system with simple roots $\Delta \sqcup \Delta^{\prime}$ is denoted by $\bar{\Phi}$.

Throughout this section, the word $Q=\left(q_{1}, \ldots, q_{r}\right)$ will denote a word in $S, \pi$ an element of $W$, and $I \subset[r]$ a facet of the subword complex $\mathcal{S C}(Q, \pi)$. Similarly, $Q^{\prime}=\left(q_{1}^{\prime}, \ldots, q_{r^{\prime}}^{\prime}\right), \pi^{\prime} \in W^{\prime}$ and $I^{\prime} \subset\left[r^{\prime}\right]$ will denote their analogous for the Coxeter system $\left(W^{\prime}, S^{\prime}\right)$.

Definition 3.5 The subword complex multiplication between two tuples is defined by

$$
(W, Q, \pi, I) \cdot\left(W^{\prime}, Q^{\prime}, \pi^{\prime}, I^{\prime}\right)=\left(\bar{W}, Q Q^{\prime}, \pi \pi^{\prime}, I I^{\prime}\right),
$$

were $Q Q^{\prime}=\left(q_{1}, \ldots, q_{r}, q_{1}^{\prime}, \ldots, q_{r^{\prime}}^{\prime}\right)$ denotes the concatenation of $Q$ and $Q^{\prime}, \pi \pi^{\prime}$ is the product of $\pi$ and $\pi^{\prime}$ in $\bar{W}$, and $I I^{\prime}$ denotes the shifted facet

$$
I I^{\prime}:=\left\{k \in\left[r+r^{\prime}\right]: k=i \text { for some } i \in I \text {, or } k=i^{\prime}+r \text { for some } i^{\prime} \in I^{\prime}\right\}
$$

The map $m$ is then extended to $\mathcal{Y} \otimes \mathcal{Y}$ by linearity. We have

$$
m: k\left[Y_{n_{1}}^{m_{1}, \ell_{1}}\right] \otimes k\left[Y_{n_{2}}^{m_{2}, \ell_{2}}\right] \longrightarrow k\left[Y_{n_{1}+n_{2}}^{\max \left\{m_{1}, m_{2}\right\}, \ell_{1}+\ell_{2}}\right] .
$$




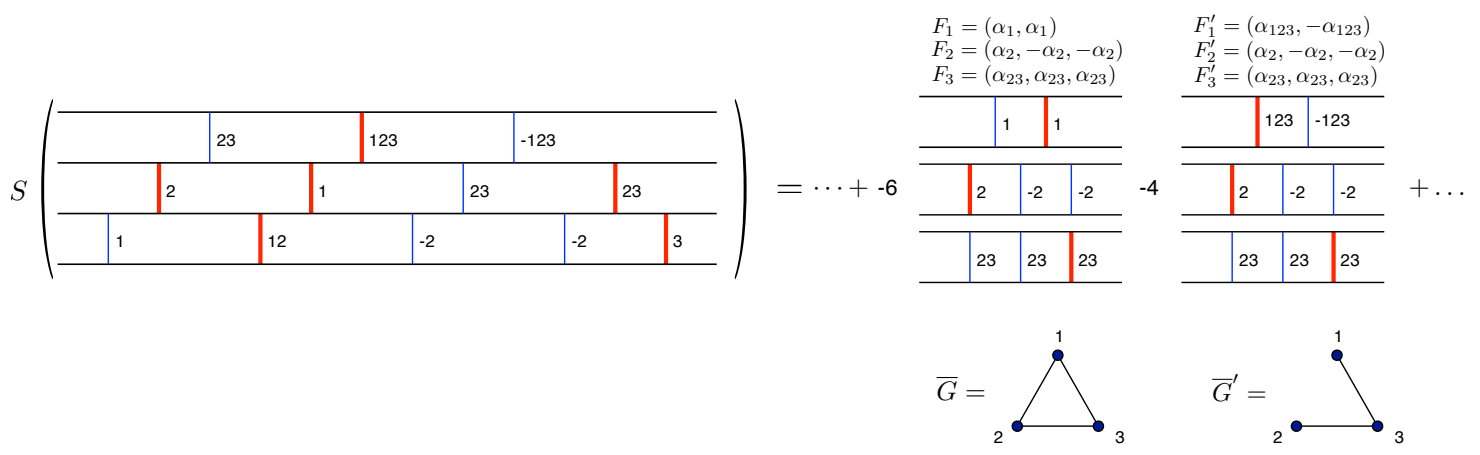

Fig. 5: Example of the cancellation free formula for the antipode. The number of acyclic orientations of the graph $\bar{G}=\bar{G}\left(\left(F_{1}, F_{2}, F_{3}\right)\right)$ is 6 , while the number of acyclic orientations of $\bar{G}^{\prime}=\bar{G}\left(\left(F_{1}^{\prime}, F_{2}^{\prime}, F_{3}^{\prime}\right)\right)$ is 4 .

\subsection{Hopf structure and antipode.}

Theorem 3.6 The graded vector space

$$
\mathcal{Y}:=\bigoplus_{n \geq 0} k\left[Y_{n}\right]
$$

equipped with the subword complex multiplication and comultiplication is a connected graded Hopf algebra. This Hopf algebra is commutative and cocommutative.

We have a cancelation free formula for the antipode that involves certain graph $\bar{G}(\mathcal{K})$ associated to maximal $r$-flat decompositions and its number of acyclic orientations $a(\bar{G}(\mathcal{K}))$. see ArXive1508.01465 for more details.

Theorem 3.7 For $\psi=(W, Q, \pi, I)$ we have

$$
S(\psi)=\sum_{\mathcal{K} \in \Psi^{0}(\mathcal{F} D)}(-1)^{\ell(\mathcal{K})} a(\bar{G}(\mathcal{K})) \cdot\left(W_{\mathcal{K}}, Q_{\mathcal{K}}, \pi_{\mathcal{K}}, I_{\mathcal{K}}\right)
$$

Remark 3.8 The formula in Theorem 3.7 is cancelation-free. Only maximal refinement contribute to the formula and isomorphic refinement all have the same length. But the formula may contain the same basis element more than once.

\section{Hopf algebra of $c$-clusters of finite type}

In [4], Ceballos, Labbé and Stump showed that the $c$-cluster complexes arising from the the theory of cluster algebras can be obtained as well chosen subword complexes. More precisely, the $c$-cluster complex is the subword complex associated to the word $Q=c w_{\circ}(c)$ and the longest element $\pi=w_{\circ}$, where $w_{\circ}(c)$ is the first lexicographically subword of $c^{\infty}$ that is a reduced expression of $w_{\circ}$.

Theorem 4.1 ([4, Theorem 2.2]) For any finite Coxeter group, the subword complex $\mathcal{S C}\left(c w_{\circ}(c), w_{\circ}\right)$ is isomorphic to the c-cluster complex. 
We will see below that our Hopf algebra induces a sub-Hopf algebra structure on this family of subword complexes. Let $\mathcal{C}_{n}$ be the subfamily of $Y_{n}$ corresponding to subword complexes of the form $\mathcal{S C}\left(c w_{\circ}(c), w_{\circ}\right)$.

Theorem 4.2 The graded vector space

$$
\mathcal{C}:=\bigoplus_{n \geq 0} k\left[\mathcal{C}_{n}\right]
$$

equipped with the subword complex multiplication and comultiplication is a connected graded sub-Hopf algebra of the Hopf algebra of subword complexes.

We briefly recall the definition of $c$-clusters and describe their Hopf algebra structure.

\section{1 c-clusters}

Let $W$ be a (non necessarily irreducible) finite Coxeter group and $\Phi$ be an associated root system. The $c$ cluster complex is a simplicial complex on the set of almost positive roots of $\Phi$, which was introduced by Reading in [20]. This complex generalizes the cluster complex of Fomin and Zelevinsky [10], and has an extra parameter $c$ corresponding to a Coxeter element.

Given an acyclic cluster seed $A$, the denominators of the cluster variables with respect to $A$ are in bijection with the set of almost positive roots. The variables in $A$ correspond to the negative roots, and any other variable to the positive root determined by the exponents of its denominator. Denote by $A^{c}$ an acyclic cluster seed corresponding to a Coxeter element $c$. Its associated (weighted) quiver corresponds to the Coxeter graph oriented according to $c$ : a pair $s, t \in S$ of non-commuting generators has the orientation $s \rightarrow t$ if and only if $s$ comes before $t$ in $c$. The $c$-clusters are the sets of almost positive roots corresponding to clusters obtained by mutations from $A^{c}$. These were described in purely combinatorial terms using a notion of $c$-compatibility relation by Reading in [20], and can be described purely in terms of the combinatorial models in the classical types [5, Section 7].

For the purpose of this paper, it is more convenient to consider $c$-clusters as pairs $\left(A^{c}, T\right)$, where $A^{c}$ is an acyclic cluster seed corresponding to $c$ and $T$ is any cluster obtained from $A^{c}$ by mutations. Note that this convention is more general than the original one. For example, two pairs related by rotation give the same $c$-cluster when considered as a set of almost positive roots.

The bijection in [4], relating positions in $Q_{c}=c w_{\circ}(c)$ to cluster variables and facets of $\mathcal{S C}\left(c w_{\circ}(c), w_{\circ}\right)$ to $c$-clusters, maps the position of $c_{i}$ in the prefix $c$ of $Q_{c}$ to the element of $A^{c}$ corresponding to $c_{i}$. The image of any other position is determined by rotation. The rotation in the classical types correspond to rotating the polygon in counterclockwise direction in the corresponding geometric models. The rotation in the word $Q_{c}$ maps the position of a letter $s$ in $Q_{c}$ to the position of the next occurrence of $s$ in $Q_{c}$, if possible, and to the first occurrence of $w_{\circ} s w_{\circ}$ otherwise.

\subsection{Hopf structure in the classical types}

Let $(A, T)$ be a $c$-cluster pair consisting of an acyclic cluster seed $A$ and any cluster $T$ obtained from $A$ by mutations. The multiplication of $c$-clusters is given by disjoint union, and the comultiplication is:

$$
\Delta((A, T)):=\sum_{U \subset T}\left(A_{U}, T_{U}\right) \otimes\left(A_{T \backslash U}, T_{T \backslash U}\right)
$$

where $A_{U}$ and $T_{U}$ denote the restrictions of $A$ and $T$ to $U$. The restriction $T_{U}$ of $T$ is simply equal to the restricted cluster $U$. The restriction $A_{U}$ of $A$ is not as clear since $U$ is not a subset of $A$, and turns out 
to be much more interesting. Denote by $\bar{U}$ the closure set of all cluster variables that can be obtained by mutating elements of $U$ in the cluster $T$ (excluding those in $T \backslash U$ ). To obtain $A_{U}$ from $A$ we proceed with the following rotation process: first take all the elements of $A$ that belong to the closure $\bar{U}$. Then, we consecutively rotate $A$ and take all its elements that belong to $\bar{U}$ and are compatible with all previously taken elements. The process finishes when we have taken as many elements as the cardinality of $U$. Theorem 4.2 guaranties that this process indeed finishes, and moreover, that it produces a cluster that is acyclic (corresponding to a Coxeter element $c_{F}$ ). In type $A_{n}$, clusters can be represented by triangulations of a convex $(n+3)$-gon. The restriction of a $c$-cluster and the rotation process is illustrated in Figure 6.
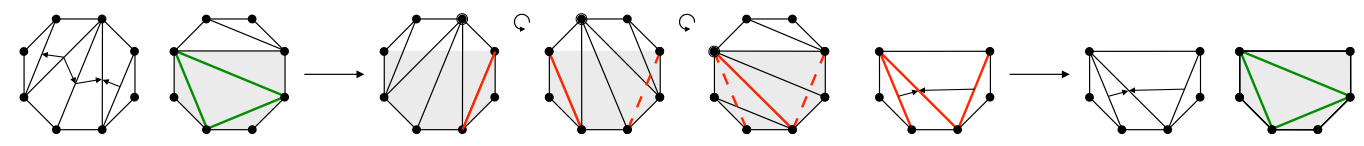

Fig. 6: Restriction of a $c$-cluster of type $A$ and rotation process restricting the cluster seed. The other types are similar.

\section{Acknowledgements}

We are especially grateful to Carolina Benedetti, Bruce Sagan, Nathan Reading, Christophe Hohlweg, Vincent Pilaud, Salvatore Stella and Jean-Philippe Labbé for helpful discussions and remarks. We also thank the Banting Postdoctoral Fellowships program of the government of Canada and York University for their support on this project.

\section{References}

[1] Marcelo Aguiar, Nantel Bergeron, and Frank Sottile. Combinatorial hopf algebras and generalized dehn-sommerville relations. Compositio Math., 142:1-30, 2006.

[2] Carolina Benedetti and Bruce Sagan. Antipodes and involutions. Preprint, arXiv:1410.5023, 2014.

[3] Nantel Bergeron, Cesar Ceballos, and Jean-Philippe Labbé. Fan realizations of type $a$ subword complexes and multi-associahedra of rank 3. Discrete \& Computational Geometry, 54(1):195-231, 2015.

[4] Cesar Ceballos, Jean-Philippe Labbé, and Christian Stump. Subword complexes, cluster complexes, and generalized multi-associahedra. J. Algebraic Combin., 39(1):17-51, 2014.

[5] Cesar Ceballos and Vincent Pilaud. Denominator vectors and compatibility degrees in cluster algebras of finite type. Transactions of the American Mathematical Society, 367(2):1421-1439, 2015.

[6] Matthew Dyer. Reflection subgroups of Coxeter systems. Journal of Algebra, 135(1):57-73, 1990.

[7] Laura Escobar. Brick manifolds and toric varieties of brick polytopes. Preprint, arXiv:1404.4671, 2014.

[8] Laura Escobar and Karola Mészáros. Subword complexes via triangulations of root polytopes. Preprint, arXiv:1502.03997, 2015. 
[9] Sergey Fomin and Andrei Zelevinsky. Cluster algebras. II. Finite type classification. Invent. Math., 154(1):63-121, 2003.

[10] Sergey Fomin and Andrei Zelevinsky. $Y$-systems and generalized associahedra. Ann. of Math. (2), 158(3):977-1018, 2003.

[11] Christophe Hohlweg, Carsten E. M. C. Lange, and Hugh Thomas. Permutahedra and generalized associahedra. Adv. Math., 226(1):608-640, 2011.

[12] Brandon Humpert and Jeremy L. Martin. The incidence hopf algebra of graphs. arXiv:1012.4786 To appear in SIAM J. Discrete Math, 2014.

[13] Jakob Jonsson. Generalized triangulations and diagonal-free subsets of stack polyominoes. J. Comb. Theory, Ser. A, 112(1):117-142, 2005.

[14] Allen Knutson and Ezra Miller. Subword complexes in Coxeter groups. Adv. Math., 184(1):161-176, 2004.

[15] Allen Knutson and Ezra Miller. Gröbner geometry of Schubert polynomials. Ann. Math., 161(3):1245-1318, 2005.

[16] Folkert Müller-Hoissen, Jean Marcel Pallo, and Jim Stasheff, editors. Associahedra, Tamari Lattices and Related Structures. Tamari Memorial Festschrift, volume 299 of Progress in Mathematics. Springer, New York, 2012.

[17] Vincent Pilaud and Michel Pocchiola. Multitriangulations, pseudotriangulations and primitive sorting networks. Discrete Comput. Geom., 48(1):142-191, 2012.

[18] Vincent Pilaud and Francisco Santos. The brick polytope of a sorting network. European J. Combin., 33(4):632-662, 2012.

[19] Vincent Pilaud and Christian Stump. Brick polytopes of spherical subword complexes and generalized associahedra. 1-61, 2015. Adv. Math., 276:1-61, 2015.

[20] Nathan Reading. Clusters, Coxeter-sortable elements and noncrossing partitions. Trans. Amer. Math. Soc., 359(12):5931-5958, 2007.

[21] Günter Rote, Francisco Santos, and Ileana Streinu. Expansive motions and the polytope of pointed pseudo-triangulations. In B. Aronov, S. Basu, J. Pach, and M. Sharir, editors, Discrete and Computational Geometry, The Goodman-Pollack Festschrift, volume 25 of Algorithms Combin., pages 699-736. Springer, Berlin, 2003.

[22] Salvatore Stella. Polyhedral models for generalized associahedra via coxeter elements. Journal of Algebraic Combinatorics, pages 1-38, 2012.

[23] Christian Stump, Hugh Thomas, and Nathan Williams. Cataland: Why the Fuss? Preprint, arXiv:1503.00710, 2015. 